\title{
royectos de Tutela del Patrimonio Subacuático realizados por el Centro de Arqueología Subacuática
}

La presentación, el pasado mes de julio, del Balneario de la Palma y el Real, en Cádiz, como sede del Centro de Arqueología Subacuática (CAS) no debe ser entendida como el punto de partida para las actividades de este Centro, sino sólo como el momento tan esperado de disponer de unas instalaciones rehabilitadas para tal fin.

Aún contando con unos medios humanos y económicos reducidos, ya en el año 1997 se iniciaron diversas labores encaminadas a crear las bases sobre las que se desarrollaría el futuro Centro. Por una parte se co- menzó a trabajar en la elaboración de una Carta de Riesgo Antrópico para el litoral andaluz, cuyos primeros resultados se exponen en un artículo aparte dentro de este Dossier. Así mismo se han ido planteando varios proyectos, unos ya están en marcha y otros inician su andadura en el presente año, enfocados a la protección, investigación histórico-arqueológica, desarrollo tecnológico, conservación-restauración, etc., los cuales se van a presentar a continuación mediante unas fichas en las que se han intentado extractar los fines y objetivos de los mismos.
Centro de Arqueología Subacuática del IAPH

\section{DENOMINACIÓN: Proyecto Baelo Claudia1}

\section{TIPO DE INTERVENCIÓN: Proyecto de investigación en el sector meridional del Conjunto arqueológico de Baelo Claudia}

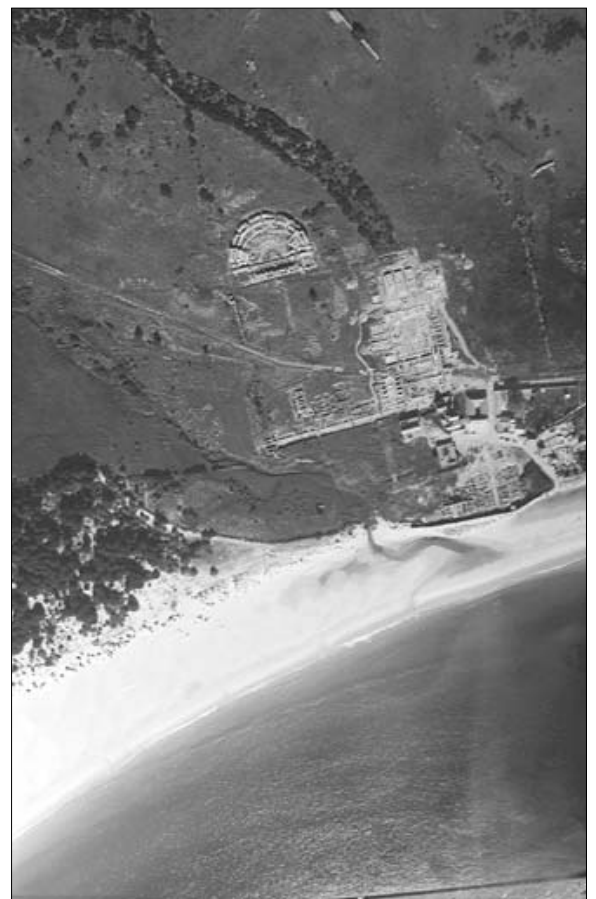

Coincidiendo con el plan de mejora de las instalaciones del Conjunto Arqueológico de Baelo Claudia planteado por la Consejería de Cultura de la Junta de Andalucía, desde el Instituto Andaluz del Patrimonio Histórico (IAPH) y el propio Conjunto Arqueológico, se ha diseñado un proyecto de investigación aplicada para actuar tanto en tierra como en el medio subacuático.

El estado actual de documentación e información sobre Baelo Claudia hace necesario, para favorecer una adecuada gestión y tutela de sus bienes patrimoniales, la detección y el conocimiento de aquellos restos arqueológicos que puedan estar localizados en el sector meridional de la antigua ciudad romana, así como la identificación de los principales elementos urbanísticos de ésta. En este sentido, el presente proyecto se enmarca dentro de las medidas cautelares necesarias para la protección y conservación material, paisajística y ambiental de la zona y el entorno del Conjunto Arqueológico.

Para cubrir los objetivos planteados se ha elaborado un plan de trabajo multidisciplinar en el que participan activamente tanto el propio Conjunto Arqueológico como el IAPH por medio de dos de sus Centros, el de Intervención y el de Arqueología Subacuática, contándose además con la colaboración de técnicos en geología y dinámica litoral de la Facultad de Ciencias del Mar de la Universidad de Cádiz. En él, además de abordarse la investigación para la delimitación (mediante técnicas geofísicas y prospecciones arqueológicas intensivas) de la zona más meridional del yacimiento, tanto en tierra como en el mar, se contempla la realización de un estudio geomorfológico y paleopaisajístico de las condiciones de la ensenada, al objeto de platear una síntesis interpretativa sobre la evolución ambiental de la misma. 
DENOMINACIÓN: Proyecto Trafalgar

\section{TIPO DE INTERVENCIÓN: Proyecto de investigación histórica-arqueológica}

El tratado de Amiens (25-III- | 802) devolvió a España la paz, tras unos años de guerras marítimas. Pero esta paz no fue muy duradera y una serie de hechos hostiles consumados por los barcos británicos, sin que hubiera existido una ruptura previa, produjeron de nuevo el estado de guerra entre ambas monarquías. Este enfrentamiento se va a materializar en la denominada BATALLA DE TRAFALGAR, combate naval mantenido el 21 de Octubre de 1805 entre la flota aliada hispano-francesa y la flota británica en aguas del Atlántico, frente a las costas españolas, en el cabo de Trafalgar.

Esta batalla ha sido motivo de numerosos estudios, desde el año 1805 hasta la actualidad, con un carácter puramente histórico, careciendo en todo momento de una investigación arqueológica que viniera a completar estos trabajos.

La presencia de restos arqueológicos localizados por azar en diferentes puntos del litoral gaditano y onubense - especialmente cañones-, así como las noticias de la existencia de determinados naufragios de barcos, supuestamente pertenecientes a las escuadras que intervinieron en la batalla, hace necesaria la realización de un proyecto que permita, mediante una metodología arqueológica y unas técnicas específicas, confirmar la existencia y cronología de aquellos hallazgos que tradicionalmente se han vinculado al combate de Trafalgar.

De esta forma, y para analizar los hechos desde un punto de vista arqueológico, se plantea el presente proyecto que tiene como objetivo principal el análisis, estudio y evaluación de los restos sumergidos como consecuencia de la batalla de Trafalgar así como del temporal que se desencadenó el día después, buscando asimismo establecer las medidas necesarias para una correcta investigación, protección, conservación y difusión de una parte importante de nuestro pasado histórico.






\section{TIPO DE INTERVENCIÓN: Proyecto experimental para la protección y puesta en valor del patrimonio arqueológico sumergido.}

El Proyecto Tarifa se enmarca dentro de las directrices de gestión cultural del patrimonio señaladas en el $\|^{\circ}$ Plan General de Bienes Culturales (1996-2000) de la Consejería de Cultura de la Junta de Andalucía y el Plan Económico para Andalucía: Horizonte 2000. En esta línea pretende buscar nuevas vías para integrar y hacer compatible el desarrollo sectorial y regional con la protección del patrimonio arqueológico subacuático, adoptando para ello medidas con clara visión de descentralización y toma de protagonismo a escala local. En suma, busca lograr la integración de cuatro elementos diferentes pero interrelacionados: protección del patrimonio arqueológico subacuático, demanda turística, oferta deportiva y riqueza de los espacios naturales subacuáticos.

En cuanto a sus objetivos, el trabajo se diseña como una primera fase experimental, a nivel microespacial, de un proyecto de protección integral más ambicioso en el tiempo y el espacio para investigar en la mejora de las técnicas y el método a aplicar en la protección de nuestro patrimonio cultural sumergido. Como marco de aplicación del mismo se ha elegido Tarifa y su entorno, por darse en él tres premisas básicas para su ejecución: una gran riqueza patrimonial subacuática, extensiva a todo el área del Estrecho de Gibraltar; un alto índice de expolio del patrimonio sumergido y una intensa actividad deportiva suba-

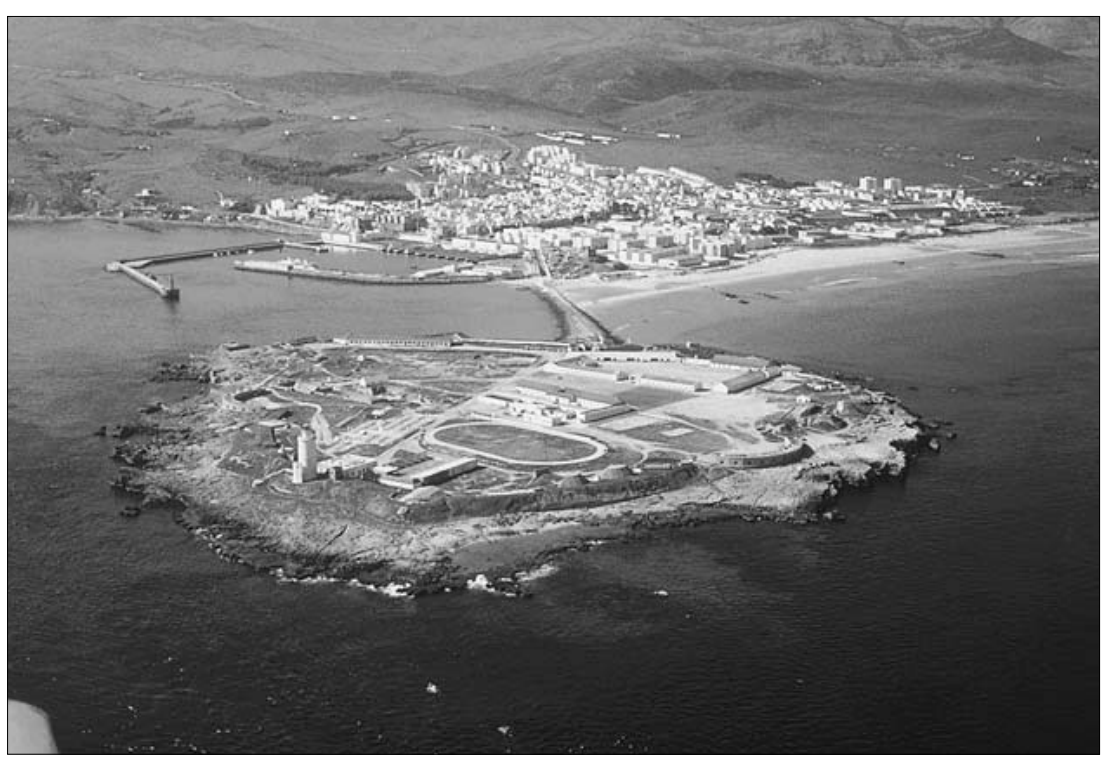
cuática encauzada a través unos pocos clubes de buceo.

Hasta el presente, ningún proyecto se ha puesto en marcha específicamente para atender a la protección del patrimonio arqueológico subacuático y luchar contra el expolio. Con éste se pretende, además de documentar en profundidad la gran riqueza arqueológica de la zona, e investigar en la mejora del método y las técnicas empleados para su protección, implicar en estas labores a los diferentes sectores sociales vinculados a la actividad del buceo, concienciándolos para ello sobre la gran fragilidad de este patrimonio, el fuerte daño que el expolio produce y las grandes posibilidades generadoras de recursos económicos mediante una correcta puesta en valor de estos bienes. 
DENOMINACIÓN: Proyecto Bajos al NW de la ciudad de Cádiz

\section{TIPO DE INTERVENCIÓN: Proyecto de investigación arqueológica}

La zona conformada por los bajos rocosos que pueblan las aguas en el sector al NW de la ciudad de Cádiz, constituye un área que a lo largo de la Historia ha presentado grandes dificultades para la navegación, especialmente a las embarcaciones propulsadas a vela y en condiciones atmosféricas adversas. Estas circunstancias, unidas a la relevancia que el entorno de la bahía ha tenido a lo largo de la historia en el comercio y tráfico marítimo, se han visto reflejadas en el enorme potencial arqueológico que goza la mencionada zona.

Entre los fondos del Museo de Cádiz se localizan un elevado número de piezas arqueológicas -ánforas, jarras, terracotas, quemaperfumes, cañones, forros de barcos, anclas, etc.- con una cronología que va desde finales del siglo VII a. C. a la actualidad, procedentes de este área y que en su mayoría se deben a hallazgos casuales. Las intervenciones arqueológicas realizadas hasta la fecha se han desarrollado de manera aislada y careciendo, en muchos casos, de una metodología arqueológica clara y precisa que permitiese establecer una correcta definición espacial y tipológica de los yacimientos localizados.

A la vista de estos antecedentes se ha elaborado un proyecto arqueológico con el que se persiguen, fundamentalmente, tres objetivos: por una parte, profundizar en el conocimiento de estos yacimientos, para lo cual se revisarán, actualizarán y sistematizarán los estudios de materiales; identificar y localizar los yacimientos, mediante unas prospecciones visuales y geofísicas, planteándose esta última si fuera oportuno; así como elaborar una síntesis interpretativa con toda la información obtenida, a fin de, por una parte, llevar a cabo una reconstrucción histórica de esta zona, y, por otra poder establecer los mecanismos necesarios para, en el marco de la legislación de patrimonio en vigor, realizar una correcta protección y conservación de los mismos.

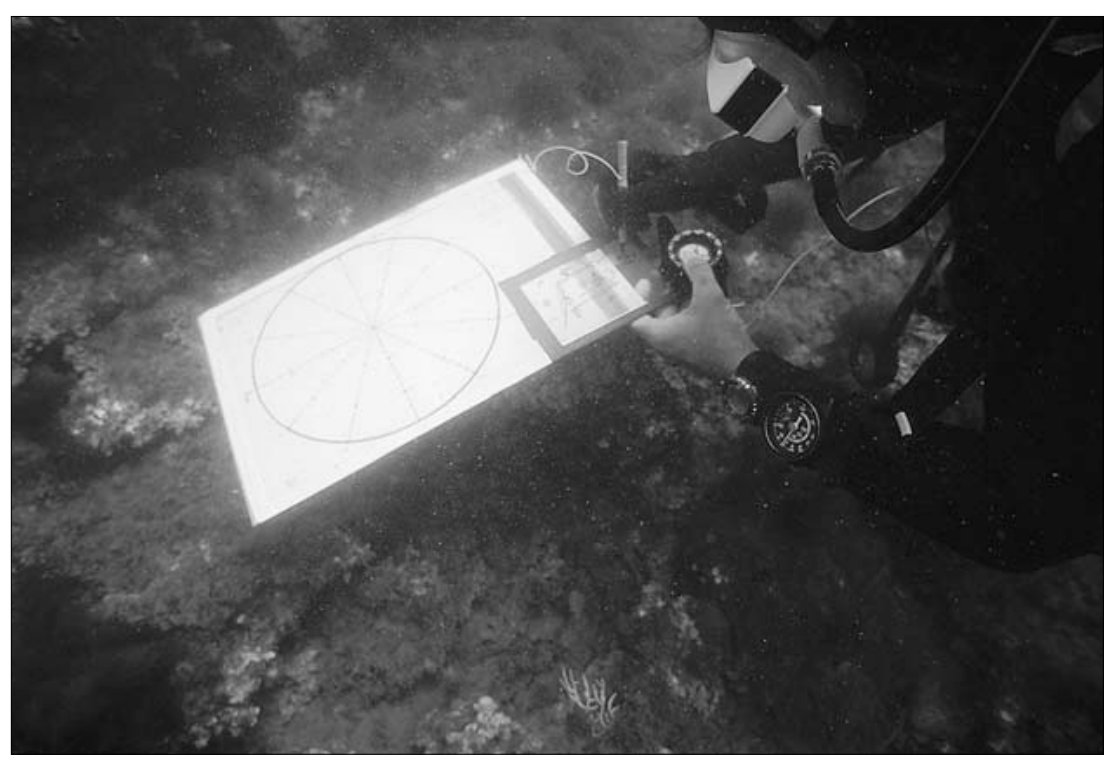




\section{DENOMINACIÓN: La prospección geofísica como técnica aplicada a la identificación y protección del patrimonio arqueológico subacuático}

\section{TIPO DE INTERVENCIÓN: Proyecto de investigación e innovación tecnológica}

La posición de la Arqueología como ciencia puente entre los estudios de Humanidades, por su finalidad, y los estudios de Ciencias y técnicas aplicadas, de las que metodológicamente cada día depende en mayor medida, ha dado lugar a que las técnicas de geofísica y teledetección hayan pasado a ser una herramienta habitual en las investigaciones arqueológicas.

La prospección arqueológica subacuática está fuertemente condicionada por el medio en el que se realiza. Así es necesario superar problemas de corrientes, falta de visibilidad, profundidad a la que se trabaja, etc. Tanto para paliar estos inconvenientes como para poder aumentar la superficie objeto de estudio, en los ochenta comenzaron a aplicarse, en la investigación arqueológica, sistemas de prospección geofísica marina: sonar de barrido lateral, penetradores de fondos, magnetómetros, detectores de metales, robots, etc. Aunque se esperaban unos resultados espectaculares, éstos fueron escasos y totalmente puntuales, de ahí la necesidad de plantear un Proyecto de Investigación encaminado a la experimentación sistemática, con objeto de planificar y poner en marcha el estudio y la innovación tecnológica para perfeccionar el conocimiento, los criterios, métodos y técnicas aplicadas a la protección, intervención y conservación del patrimonio arqueológico subacuático.

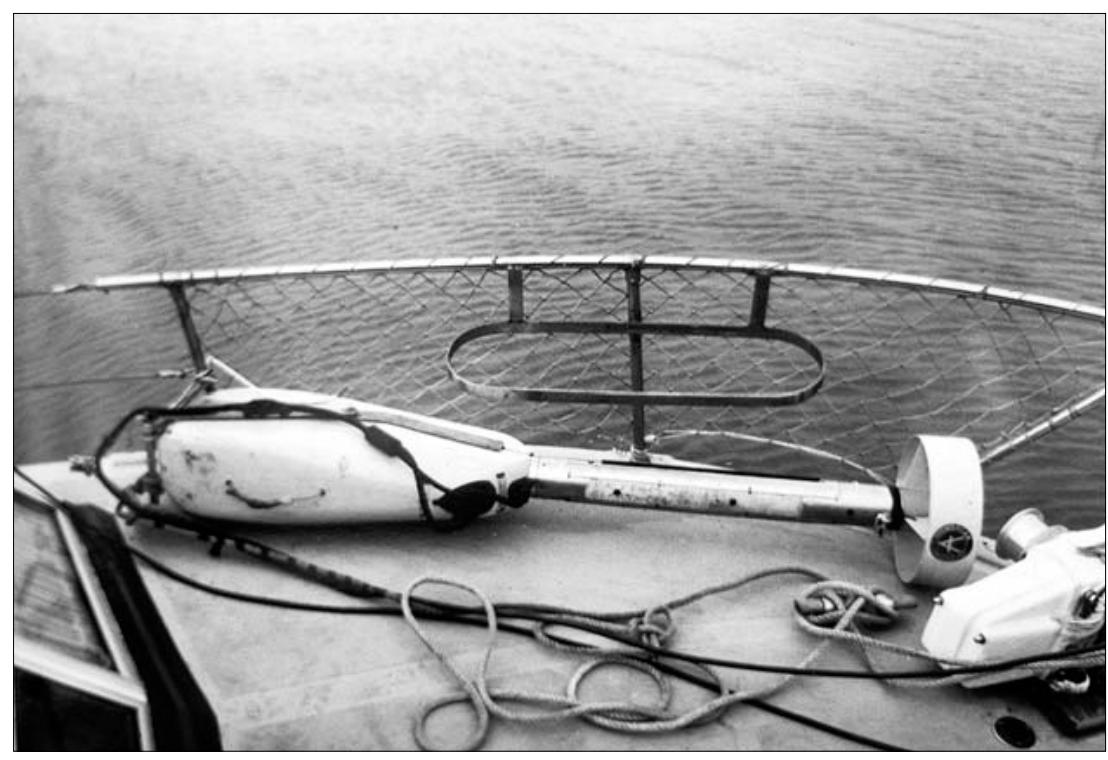

Esta debe ser la vía adecuada para llegar a conclusiones válidas que permitan establecer qué técnicas de prospección geofísica y en qué condiciones pueden ser consideradas como nuevas herramientas para la identificación y protección del PAS. 
DENOMINACIÓN: La dinámica litoral y su aplicación a la localización y conservación del Patrimonio Arqueológico Subacuático

\section{TIPO DE INTERVENCIÓN: Proyecto I+Dt sobre cambios de la línea de costa}

Las nuevas líneas de investigación abiertas en el campo de la Historia y la Arqueología, han puesto de manifiesto la necesidad de contar con estudios de tipo geoarqueológicos y paleobambientales que permitan realizar una reconstrucción de la evolución paisajística a lo largo de los siglos. En este sentido, la ejecución de proyectos multidisciplinares está haciendo posible conocer las fuertes transformaciones que el paisaje, especialmente en la zona litoral, ha venido sufriendo como consecuencia de la incidencia de agentes tanto naturales como antrópicos. Ello ha permitido saber que, no en pocos casos, se ha producido una descontextualización de determinados elementos de nuestro patrimonio histórico-arqueológico del ambiente natural para el que fueron creados, lo que ha provocado en parte la pérdida de su auténtico significado histórico.

Este fenómeno, común a muchas zonas del litoral andaluz, viene producido unas veces por la colmatación ocasionada por el aporte fluvial o por la propia acción antrópica, lo que permite actualmente localizar tierra adentro barcos o zonas portuarias, otras veces es la influencia de la erosión marina y de otros eventos naturales la causa de encontrar bajo agua restos propios de un patrimonio terrestre.

Como marco para la aplicación de este proyecto se ha escogido la Bahía de Cádiz, entre otros motivos por que, desde hace años, son numerosos los investigadores que han estudiado la paleotopografía de la Bahía y su evolución, pero desde una perspectiva carente de la multidisciplinariedad que ahora se plantea con la colaboración de la Facultad de Ciencias del mar de la Universidad de Cádiz.

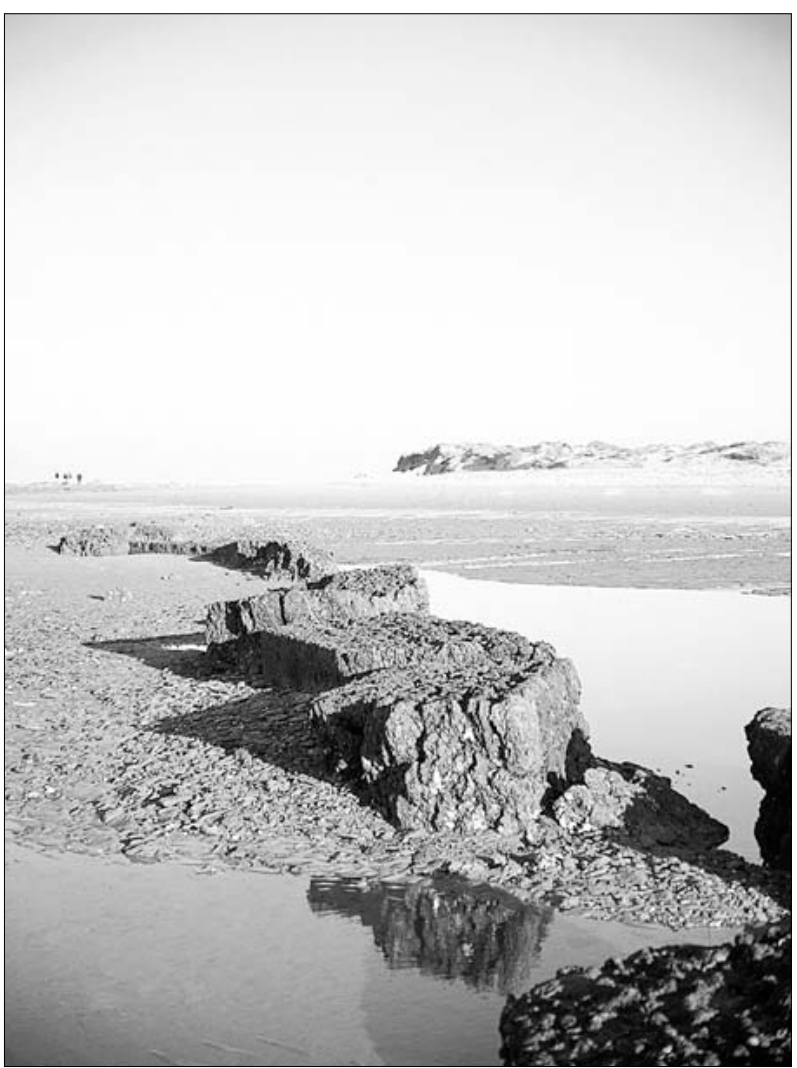




\section{TIPO DE INTERVENCIÓN: Proyecto de investigación metodológica y técnica para la conservación} preventiva en el Patrimonio Arqueológico Subacuático

La conservación preventiva se ha definido como la intervención urgente sobre las causas de alteración, de un modo indirecto sobre el medio, o directo sobre el objeto, ante un riesgo inmediato de pérdida de información. Este proyecto tiene como objetivo establecer y desarrollar un cuerpo de actuaciones de conservación preventiva aplicada al Patrimonio Arqueológico Subacuático (P.A.S.).

La inexistencia de una metodología global y sistemática que atienda la conservación preventiva del P.A.S. en su doble consideración de: $1 /$ objeto de investigación científica y $2 /$ objeto de protección cultural, justifica la redacción de un programa específico. Éste será de carácter multidisciplinar ya que plantea la coordinación de técnicos y profesionales especializados en: arqueología y conservación subacuática, documentación, difusión, gestión del patrimonio y análisis instrumental del medio marino.

En la práctica, se establecen dos fases de actuación: I/ conservación preventiva de yacimientos arqueológicos y $2 /$ conservación preventiva en intervenciones arqueológicas.

La conservación preventiva de yacimientos comienza con la investigación documental que comprende: localización geográfica y valoración arqueológica. Paralelamente, se analizan los factores de riesgo para la conservación del yacimiento; parámetros físico-químicos ( $\mathrm{T}^{\mathrm{a}}, \mathrm{pH}, \mathrm{O} 2$, Eh, corrientes, edafología, presión...), biológicos y antrópicos (obras públicas, expolio, actividades náuticas). La medición periódica de los valores obtenidos permitirá establecer un diagnóstico sobre el tratamiento de protección requerido para cada yacimiento. Entre las medidas de actuación pasiva están: la protección


legal / administrativa, la protección física / material, la formación básica del personal no-conservador y la dinamización socio-cultural.

La excavación sistemática del yacimiento, realizada por el equipo de intervención arqueológica, es una medida "activa" que planteará un desarrollo específico de conservación preventiva. Para ello se requiere una planificación coordinada de las actuaciones de investigación arqueológica y conservación.

El diseño de este plan deberá atender tanto a los recursos materiales como a la situación del yacimiento considerando: I/ datos arqueológicos (tipología, datación, área de dispersión, concentración y volumen), 2/ factores de alteración intrínsecos (parámetros físicos, químicos, biológicos) y 3 / factores externos (distancia del laboratorio, riesgo antrópico, accesibilidad).

Su aplicación deberá poner a punto todos los sistemas de conservación preventiva para preservar la integridad física de los objetos y el yacimiento durante el proceso de recuperación sistemática de los materiales arqueológicos. En su desarrollo se atenderán todos los aspectos funcionales derivados de dicha actividad: excavación, extracción, transporte, acondicionamiento, registro, estabilización, almacenamiento y exposición. 


\section{DENOMINACIÓN: Aplicación del método electrolítico de conservación para artefactos de hierro procedentes del mar}

\section{TIPO DE INTERVENCIÓN: Proyecto de optimización del método electrolítico de conservación}

Los materiales arqueológicos procedentes de yacimientos subacuáticos presentan uno de los más difíciles problemas a los que se enfrentan los laboratorios de conservación. El hecho de haber permanecido sumergidos durante prolongados espacios de tiempo, les confiere unas propiedades especiales. Además estos materiales han alcanzado un estado de equilibrio con el medio, y cuando se extraen éste se rompe, dando lugar a un acelerado proceso de deterioro.

Tras un análisis de los diversos materiales de procedencia subacuática se observa que el hierro es, de todos los metales rescatados del mar, el más abundante y el que presenta mayores problemas. Cuando éste procede de yacimientos arqueológicos terrestres se corroe mediante un proceso electroquímico. En el mar este proceso se acelera como consecuencia del contenido en sales del agua, siendo este proceso cinco veces más rápido que en tierra.

En una intervención arqueológica subacuática, una amplia variedad de objetos de hierro puede ser extraída del mar (clavos, balas de cañón, cañones, anclas, etc.) que, en su mayoría, no son tratados o no lo son adecuadamente debido a la inexistencia de laboratorios nacionales capaces de llevar a cabo su conservación con resultados satisfactorios.

Para solventar este problema, el Departamento de Conservación del C.A.S. proyecta el montaje de un laboratorio especializado en la conservación/consolidación de artefactos de hierro de procedencia marina. Para ello, tomando como punto de partida las experiencias realizadas por D.L. Hamilton en la Universidad de Texas, se pretende utilizar métodos electrolíticos, adaptándolos a nuestras necesidades y estudiando en qué forma influyen las diferentes variables del proceso.

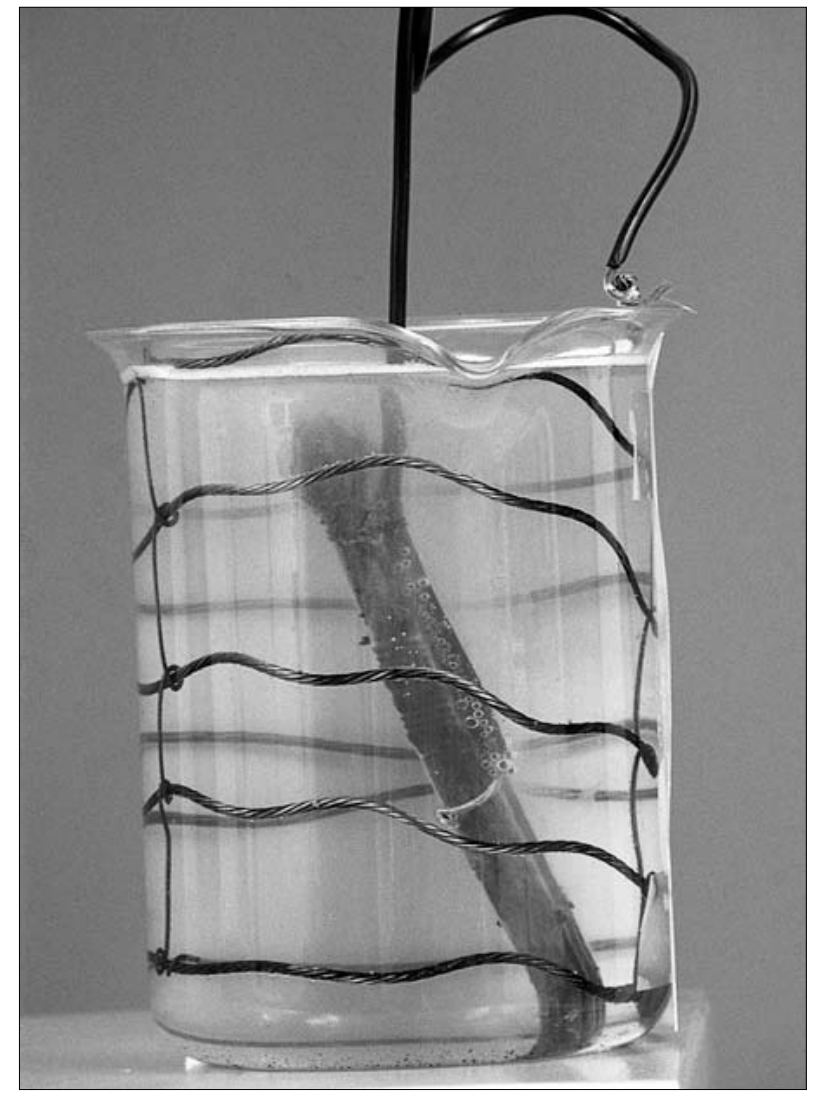

